\title{
A sequence variant on $17 q 21$ is associated with age at onset and severity of asthma
}

Eva Halapi ${ }^{1}$, Daniel F Gudbjartsson ${ }^{1}$, Gudrun M Jonsdottir ${ }^{1}$, Unnur S Bjornsdottir ${ }^{1,2}$, Gudmar Thorleifsson ${ }^{1}$, Hafdis Helgadottir ${ }^{1}$, Carolyn Williams ${ }^{3,4,5}$, Gerard H Koppelman ${ }^{6}$, Andrea Heinzmann ${ }^{7}$, H Marike Boezen ${ }^{8}$, Aslaug Jonasdottir ${ }^{1}$, Thorarinn Blondal ${ }^{1}$, Sigurjon A Gudjonsson ${ }^{1}$, Adalbjorg Jonasdottir ${ }^{1}$, Theodora Thorlacius ${ }^{1}$, Amanda P Henry ${ }^{9}$, Janine Altmueller ${ }^{10}$, Marcus Krueger ${ }^{7}$, Hyoung Doo Shin ${ }^{11,12}$, Soo-Taek Uh ${ }^{13}$, Hyun Sub Cheong ${ }^{12}$, Brynja Jonsdottir ${ }^{14}$, Bjorn R Ludviksson ${ }^{2,15}$, Dora Ludviksdottir ${ }^{16}$, David Gislason ${ }^{16}$, Choon-Sik Park ${ }^{17}$, Klaus Deichmann ${ }^{7}$, Philip J Thompson ${ }^{3,4,5}$, Matthias Wjst ${ }^{18,19}$, Ian P Hall ${ }^{9}$, Dirkje S Postma ${ }^{20}$, Thorarinn Gislason ${ }^{2,16}$, Augustine Kong ${ }^{1}$, Ingileif Jonsdottir ${ }^{1,2,15}$, Unnur Thorsteinsdottir ${ }^{\star, 1,2}$ and Kari Stefansson ${ }^{\star, 1,2}$

A sequence variant (rs7216389-T) near the ORMDL3 gene on chromosome 17q21 was recently found to be associated with childhood asthma. We sought to evaluate the effect of rs7216389-T on asthma subphenotypes and its correlation with expression levels of neighboring genes. The association of rs7216389-T with asthma was replicated in six European and one Asian study cohort ( $N=4917$ cases $N=34589$ controls). In addition, we found that the association of rs7216389-T was confined to cases with early onset of asthma, particularly in early childhood (age: $0-5$ years $0 R=1.51, P=6.89 \cdot 10^{-9}$ ) and adolescence (age: $14-17$ years $O R=1.71, P=5.47 \cdot 10^{-9}$ ). A weaker association was observed for onset between 6 and 13 years of age $(O R=1.17, P=0.035)$, but none for adult-onset asthma $(O R=1.07, P=0.12)$. Cases were further stratified by sex, asthma severity and atopy status. An association with greater asthma severity was observed among early-onset asthma cases $(P=0.0012)$, but no association with sex or atopy status was observed among the asthma cases. An association between sequence variants and the expression of genes in the 17q21 region was assessed in white blood cell RNA samples collected from Icelandic individuals $(n=743)$. rs7216389 associated with the expression of GSDMB and ORMDL3 genes. However, other sequence variants showing a weaker association with asthma compared with that of rs7216389 were more strongly associated with the expression of both genes. Thus, the contribution of rs7216389-T to the development of asthma is unlikely to operate only through an impact on the expression of ORMDL3 or GSDMB genes.

European Journal of Human Genetics (2010) 18, 902-908; doi:10.1038/ejhg.2010.38; published online 7 April 2010

Keywords: childhood asthma; single-nucleotide polymorphism; expression; ORMDL3; GSDMB

\section{INTRODUCTION}

Multiple environmental and hereditary factors affect the development of asthma. ${ }^{1-3}$ More than 200 genes have been reported to harbor sequence variants that associate with asthma or related phenotypes, but few have been robustly replicated. ${ }^{4,5}$ In a recent genome-wide association study of asthma, the sequence variant rs7216389-T on chromosome $17 \mathrm{q} 21$ was shown to associate with the risk of childhood asthma. $^{6}$ rs7216389 is located in an intron of the GSDMB gene (encoding the gasdermin-like protein), but its association with asthma was originally linked to the expression of the nearby ORMDL3 gene (encoding the ORM1-like 3 protein). Subsequent reports have provided further support for the association of this 17q21 locus with childhood ${ }^{7-11}$ and persistent asthma, ${ }^{12}$ but the mechanisms underlying this effect remain unclear.

Asthma is a heterogeneous disease with respect to age at onset, clinical presentation, disease progression and therapeutic response. ${ }^{3}$ To investigate the phenotype-genotype relationship of the sequence variant at $17 \mathrm{q} 21$ with asthma, we genotyped tagging SNPs at this locus (including rs7216389) in samples obtained from Iceland, Australia, The Netherlands, Germany, the United Kingdom and Korea with

\footnotetext{
${ }^{1}$ deCODE Genetics Inc., Reykjavik, Iceland; ${ }^{2}$ Faculty of Medicine, University of Iceland, Reykjavik, Iceland; ${ }^{3}$ The Lung Institute of Western Australia, Nedlands, Australia; ${ }^{4}$ The Western Australian Institute of Medical Research, Perth, Australia; ${ }^{5}$ The Centre for Asthma, Allergy and Respiratory Research, University of Western Australia, Perth, Australia; ${ }^{6}$ Department of Pediatric Pulmonology and Pediatric Allergology, Beatrix Children's Hospital, University Medical Center Groningen, University of Groningen, Groningen, The Netherlands; ${ }^{7}$ Centre of Pediatrics and Adolescent Medicine, University of Freiburg, Freiburg, Germany; ${ }^{8}$ Department of Epidemiology, University Medical Center Groningen, University of Groningen, Groningen, The Netherlands; ${ }^{9}$ Division of Therapeutics, University Hospital of Nottingham, Queen's Medical Centre, Nottingham, UK; ${ }^{10}$ Cologne Center for Genomics (CCG), University of Cologne, Cologne, Germany; ${ }^{11}$ Laboratory of Genomic Diversity, Department of Life Science, Sogang University, Seoul, Korea; ${ }^{12}$ Department of Genetic Epidemiology, SNP Genetics Inc., Seoul, Korea; ${ }^{13}$ Division of Allergy and Respiratory Medicine, Soonchunhyang University Seoul Hospital, Seoul, Korea; ${ }^{14}$ Department of Internal Medicine, Landspitali University Hospital, Reykjavik, Iceland; ${ }^{15}$ Department of Immunology, Landspitali University Hospital, Reykjavik, Iceland; ${ }^{16}$ Department of Allergy, Respiratory Medicine and Sleep, Landspitali University Hospital, Reykjavik, Iceland; ${ }^{17}$ Division of Allergy and Respiratory Medicine, Soonchunhyang University, Bucheon Hospital, Bucheon, Korea; ${ }^{18}$ Institute of Lung Biology and Disease, Helmholtz Zentrum München, German Research Center for Environmental Health, Neuherberg, Germany; ${ }^{19}$ Institute of Genetic Medicine, EURAC Research, Bozen, Germany; ${ }^{20}$ Department of Pulmonology, University Medical Center Groningen, University of Groningen, Groningen, The Netherlands

*Correspondence: Dr K Stefansson or Dr U Thorsteinsdottir, Population Genomics, deCODE Genetics Inc., Sturlugata 8, Reykjavik, IS 101 , Iceland. Tel: +354 570 1900; Fax: +354 570 1903; E-mail: kstefans@decode.is or unnurth@decode.is

Received 1 May 2009; revised 13 January 2010; accepted 25 February 2010; published online 7 April 2010
} 
information on age at onset, sex, asthma severity and atopy status. We also examined the correlation of those SNPs with the expression of genes in the region around rs7216389 in white blood cells of an Icelandic sample set.

\section{METHODS}

\section{Study samples}

The samples used in this study consisted of case-control sample sets obtained from Iceland, Germany, The Netherlands, the United Kingdom, Australia and Korea, and a familial sample set from Germany (Table 1). Informed consent was obtained locally from all participants and all studies were approved by the local ethics committees. The individual sample sets have been described in detail elsewhere ${ }^{13-24}$ (see also brief cohort descriptions in Supplementary text).

For all sample sets, a diagnosis of asthma based on patient symptoms and clinical testing was given by a physician at each local clinical center on the basis of international and national asthma guidelines. Atopy status (defined by at least one positive response to allergens) as determined by skin prick testing was available for all samples, except the UK set. Asthma severity was available in the Icelandic, Australian and UK sample sets. In the Icelandic cases, asthma severity was assessed using criteria from the National Heart, Lung and Blood Institute (NHLBI) ${ }^{25}$ and GINA guidelines. ${ }^{26}$ In addition, patient charts were reviewed specifically for frequency of daytime and nocturnal symptoms and impact on daily activities, asthma-related health-care use (use of emergency care, hospitalizations and physician office visits), medication use (dose of inhaled corticosteroids) and use of add-on medications such as long- and short-acting $\beta$-agonists, systemic corticosteroids, theophylline and leukotriene antagonists. The criteria used to assess asthma severity in the Australian sample set were described previously. ${ }^{17}$ Briefly, the clinical parameters used for severity scoring included lung function (\% predicted $\mathrm{FEV}_{1}$ ), use of oral corticosteroids, daily inhaled corticosteroid dose, weekly frequency of use of rescue medication, weekly frequency of daytime symptoms, weekly frequency of nighttime wakening because of asthma, unplanned visits to a general practitioner and hospital admissions for asthma in the preceding 12 months. On the basis of national ${ }^{27}$ and NHLBI guidelines, ${ }^{25}$ patients were classified as mild, moderate or severe for each criterion and the most common classification was used as a measure of asthma severity. For the UK cohort, severity assessment was based on the British Thoracic Society guidelines. ${ }^{28}$ Steps 1-2 were considered as mild, step 3 was as considered moderate and steps $4-5$ were considered as severe asthma.

Information on age at onset of asthma and sex was available for all sample sets, except for the Germany I set. Early onset was defined as the onset of asthma at or under 18 years of age, and adult onset was defined as the onset at or over 19 years of age in all study sample sets. In Iceland, the age at onset was assessed with a questionnaire using the question 'How old were you when you had your first asthma-attack?' For the Australian and UK sample sets, the age at onset was assessed with questionnaires and a subsequent interview using the question 'How old were you when you were diagnosed with asthma?' For the Korean and Dutch sample sets, the age at onset was assessed with a questionnaire using the question 'How old were you when you first experienced asthma symptoms?' For the Germany II sample set, the age at onset was assessed with a parental interview asking for the age at occurrence of wheezing.

Total serum IgE levels were measured by UniCAP FEIA (Pharmacia Upjohn, Uppsala, Sweden).

\section{Genotyping}

All genotyping procedures of SNPs in the 17q21 region were carried out at deCODE Genetics using Hap300 bead-array (Illumina Inc., San Diego, CA, USA), TaqMan (Applied Biosystems Inc., Cheshire, UK) or Centaurus platforms (NanoGen Inc., San Diego, CA, USA). In Iceland, 1192 asthma patients and 30237 controls were genotyped with the Illumina 300K chip. An additional 456 Icelandic asthma patients and 661 Icelandic controls and all non-Icelandic samples were genotyped using either single SNP TaqMan or Centaurus platforms. The SNPs used in the $17 \mathrm{q} 21$ region on the Illumina $300 \mathrm{~K}$ chip passed quality filters (yield $>95 \%$, minor allele frequency $>0.01$, no distortion of Hardy-Weinberg equilibrium (HWE) in the controls $(P>1.0 \times 10-3))$. All samples had call rates above $98 \%$. The quality of TaqMan or Centaurus SNP assays was evaluated by genotyping each assay in the CEU HapMap samples and comparing the results with the publicity released HapMap data. Assays with $>1.5 \%$ mismatch rate were not used. Supplementary Table 2 contains allele frequencies and HWE $P$-values for the different sample sets.

\section{Expression analysis}

Expression levels for genes in the 17q21 region and correlation with the genotype status of 15 SNPs in the region were assessed with Human $25 \mathrm{~K}$ chip array (Agilent Technologies Inc.) as part of a separate study in Iceland ${ }^{29}$ (expression data available as GEO data set GSE7695 and GPL3991). The collection of white blood cell samples $(N=743)$, mRNA isolation and expression profiling have been described previously. ${ }^{29}$ Expression was quantified as mean logarithm $\left(\log _{10}\right)$ expression ratio (MLR), that is, expression ratios compared with background-corrected intensity values. ${ }^{29}$ The array expression data were validated by performing TaqMan gene expression assays for IKZF3 (encoding the IKAROS family zinc-finger 3 protein), GDSM1 (encoding the gasdermin 1 protein), ZPBP2 (encoding the zona pelliculada-binding protein 2), GSDMB and ORMDL3 genes using CDNA derived from the same white blood cell RNA samples from individuals tested on the Agilent chip. TaqMan assays for ZPBP2 and GDSM1 did not yield any product in white blood cells or other cDNA source tested (data not shown). TaqMan gene expression assays were also performed on mRNA from Epstein-Barr Virus

Table 1 Study populations

\begin{tabular}{|c|c|c|c|c|c|c|c|}
\hline & Iceland & Australia & The Netherlands & Korea & United Kingdom & Germany I & Germany II \\
\hline Recruitment phenotype & Asthma & Asthma & Asthma & Asthma & Asthma & Childhood/adolescent asthma & Childhood asthma \\
\hline Study design & Case-control & Case-control & Case-control & Case-control & Case-control & Case-control & $\begin{array}{l}\text { Affected } \\
\text { Sib-pairs }\end{array}$ \\
\hline$N$ cases/controls & $1648 / 30898$ & $647 / 564$ & $221 / 1564$ & $1387 / 558$ & $292 / 241$ & $307 / 560$ & $415 / 204^{a}$ \\
\hline $\begin{array}{l}\text { Male/female }{ }^{b} \\
N \text { age at onset }{ }^{b}\end{array}$ & $475 / 1173$ & $244 / 403$ & $136 / 85$ & $588 / 799$ & $116 / 176$ & $198 / 109$ & $240 / 175$ \\
\hline $\begin{array}{l}\text { Early/adult/unknown }{ }^{\mathrm{c}} \\
N \text { atopy }^{\mathrm{b}}\end{array}$ & $617 / 744 / 287$ & $370 / 226 / 51$ & $156 / 57 / 8$ & $211 / 1176 / 0$ & $138 / 60 / 94$ & $307 / 0 / 0$ & $415 / 0 / 0$ \\
\hline Atopic/nonatopic/unknown & $890 / 697 / 61$ & $539 / 106 / 2$ & $181 / 31 / 9$ & $829 / 541 / 17$ & $N A^{e}$ & $277 / 30 / 0$ & $319 / 96 / 0$ \\
\hline $\begin{array}{l}\log _{10} \text { total serum } \operatorname{lgE}^{\mathrm{b}, \mathrm{d}} \\
N \text { severity score }\end{array}$ & $1.7 \pm 0.7$ & $2.0 \pm 0.6$ & $2.0 \pm 0.6$ & $2.2 \pm 0.6$ & NA & $2.4 \pm 0.6$ & $2.3 \pm 0.7$ \\
\hline Mild/moderate/severe/unknown & $547 / 537 / 118 / 446$ & $336 / 233 / 78 / 0$ & NA & NA & 28/73/65/126 & NA & NA \\
\hline
\end{tabular}

an estimate of the number of untransmitted parental alleles were used as control alleles. bVariables related to cases only.

'Early onset=at or younger than 18 years of age; adult onset=at 19 years of age or older.

Early onset- $=$ Mean \pm s.d.
dMet availabe.

dMean \pm s.d.
eNot available. 
(EBV)-transformed B lymphocytes ${ }^{30}$ from Icelandic prostate cancer patients $(N=186)$ and from peripheral blood mononuclear cells (PBMCs) isolated from Icelandic patients with asthma $(N=182) .{ }^{31} \mathrm{cDNA}$ was synthesized using the high-capacity cDNA reverse transcriptase kit (Applied Biosystems Inc.). TaqMan expression assays were performed with the TaqMan Universal PCR Master Mix (Applied Biosystems Inc.), using PCR primers from Eurofins mwg operon (Ebersberg, Germany) and MGB TaqMan probes (Applied Biosystems Inc.) (for primer and probe sequences, see Supplementary Table 1).

\section{Statistical analysis}

To replicate the previously reported association of chromosome 17q21 SNPs with asthma, we tested their association with asthma within each population. We then explored whether the strength of association depended on the age at onset of asthma by breaking the sample sets into children, adults with early onset and adults with adult onset (Table 2). We further studied the effect of age at onset by testing the association within 2-year age bins (Figure 1). On the basis of the results of the age-stratified analysis, subsequent testing was performed separately within early- and adult-onset groups. We then examined whether the association with asthma depended on sex, asthma severity, atopy status and IgE levels.

For association analysis, we used standard likelihood ratio statistics, implemented in the NEMO software, ${ }^{32}$ to calculate two-sided $P$-values and allelespecific ORs for each individual allele, assuming a multiplicative model (the two alleles are independent, or in HWE, within the population of affected individuals). Allele frequencies were estimated by maximum likelihood and calculated directly for the observed data, and tests of differences between cases and controls were performed using a generalized likelihood ratio test. ${ }^{32}$

The sib-pairs from the Germany II sample set were analyzed assuming Mendelian inheritance under the null hypothesis. Under alternative transmission, probabilities were defined by the multiplicative risk model and allele frequencies. Untransmitted parental alleles were used as controls. The number of untransmitted parental genotypes corresponds to having genotyped 204 control individuals on an average.

A test for severity among cases was conducted by logistic regression, treating allele counts as the response variable and the severity class as an explanatory variable, encoding the severity classes as 0 (mild), 1 (moderate) and 2 (severe).

Results obtained from multiple groups were combined using a MantelHaenszel mode ${ }^{33}$ that allows for analysis incorporating disparate frequencies for alleles and genotypes for different populations, but assuming the same relative risks. Tests of heterogeneity were conducted by assuming that the allele frequencies were the same in all groups under the null hypothesis, but that each group had a different allele frequency under the alternative hypothesis.

The correlation between the expression ratios (MLR ${ }^{29}$ for the genes analyzed and genotypes of each of the SNPs in the locus was tested by regressing MLRs against the SNPs' allele count after adjustment for age and sex.

Multiple logistic regression was used to test the association of SNPs with asthma, conditional on another SNP's association with asthma. Similarly, multiple linear regression was used to test the association of an SNP with quantitative expression levels, conditional on another SNP's association with the expression levels.

\section{RESULTS}

\section{Disease association}

We replicated the previously reported association of allele $\mathrm{T}$ of rs7216389 with asthma ${ }^{6}$ in 1648 Icelandic asthma cases and 30898 Icelandic controls $\left(\mathrm{OR}(95 \% \mathrm{CI})=1.23(1.14-1.33), P=1.56 \times 10^{-7}\right)$. The effect in Iceland was weaker than the previously reported ORs of $1.45^{6}$ and $1.50^{8}$ in asthmatic patients of European descent. Previous studies were predominantly conducted in pediatric cohorts, whereas in the Icelandic sample set, the cases are adults. ${ }^{6,11}$ We therefore assessed the strength of the association with rs7216389-T stratified on age at onset of asthma, dividing the cases into early onset (before or at 18 years of age $n=617$ ) and adult onset (at or after 19 years of age $n=744$ ). Early-onset cases showed a substantially stronger association $(\mathrm{OR}(95 \% \mathrm{CI})=1.44(1.28-1.63))$ than did adult-onset cases (OR $(95 \% \mathrm{CI})=1.11(0.99-1.23))($ Table 2$)$.

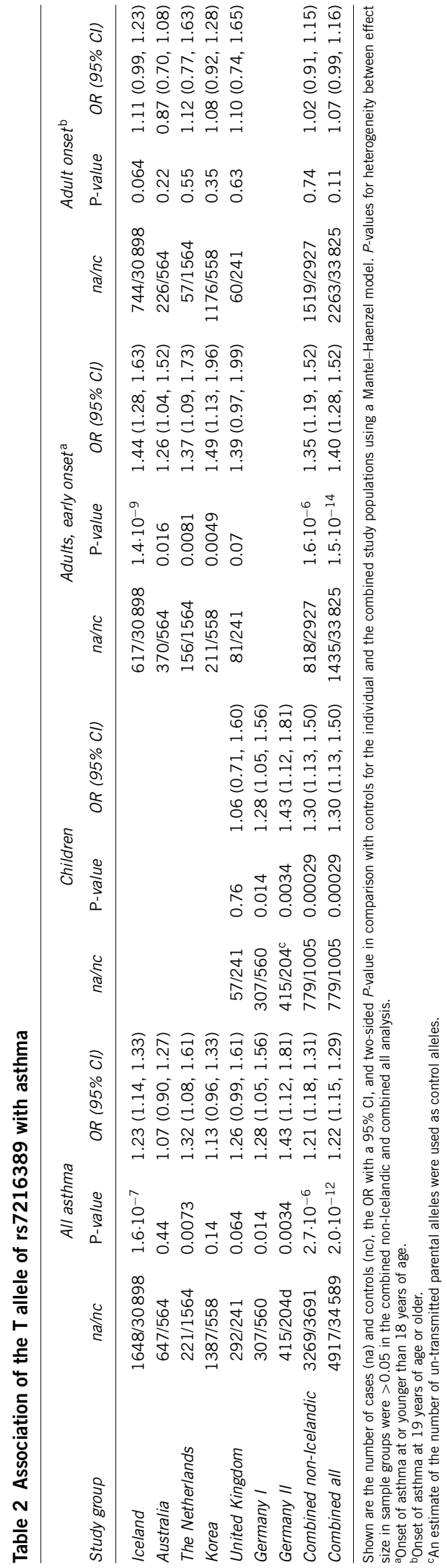




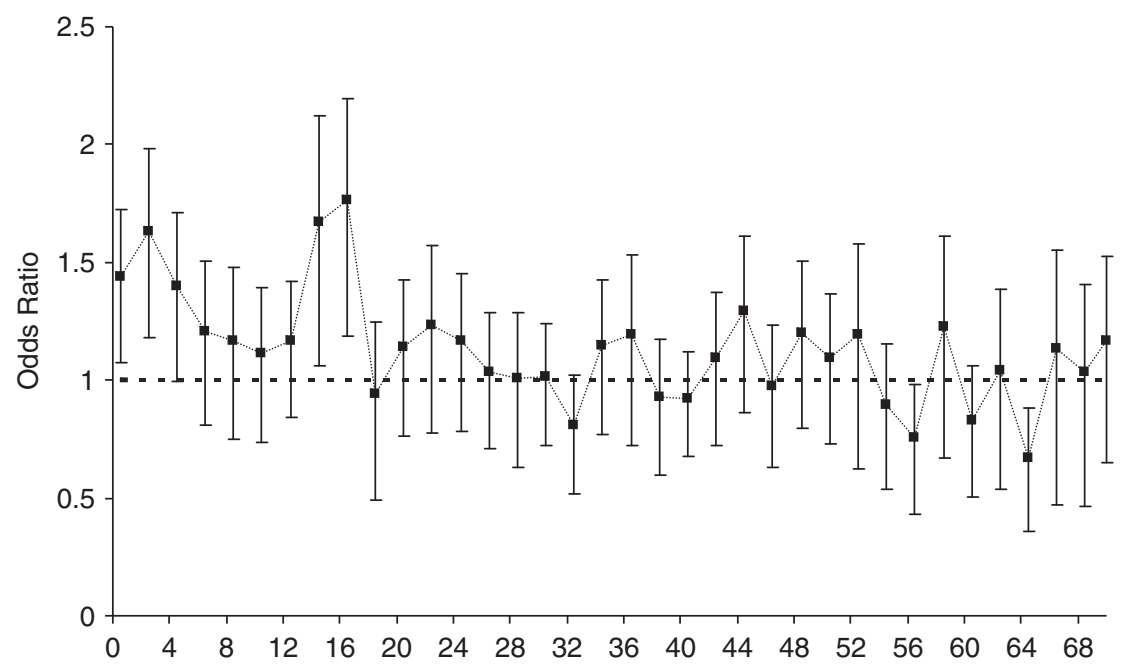

Figure 1 Association with rs7216389-T stratified by age at onset of asthma. Cases were stratified by age at onset, and association with rs7216389-T was assessed for each age bin (0-1, 2-3, 4-5, 6-7 years and so on). Cases with onset at 70 years or older were pooled into one single bin (70+). The OR and $95 \% \mathrm{Cl}$ for the mean age of each bin for the combined sample set (Iceland, Australia, United Kingdom, Korea and Germany II) are plotted.

Table 3 Association results for the T allele of rs7216389 with early onset of asthma stratified by severity ${ }^{a}$

\begin{tabular}{|c|c|c|c|c|c|c|c|c|}
\hline \multirow[b]{2}{*}{ Study group } & \multirow[b]{2}{*}{$\mathrm{N}$ control } & \multicolumn{2}{|r|}{ Mild } & \multicolumn{2}{|r|}{ Moderate } & \multicolumn{2}{|r|}{ Severe } & \multirow[b]{2}{*}{ P-value } \\
\hline & & $\mathrm{N}$ cases & OR $(95 \% \mathrm{Cl})$ & $\mathrm{N}$ cases & OR $(95 \% \mathrm{Cl})$ & $\mathrm{N}$ cases & OR $(95 \% \mathrm{Cl})$ & \\
\hline Iceland & 30898 & 200 & $1.37(1.12,1.67)$ & 196 & $1.52(1.25,1.86)$ & 27 & $2.86(1.64,4.98)$ & 0.052 \\
\hline UK & 241 & 18 & $1.40(0.71,2.77)$ & 35 & $1.61(0.97,2.67)$ & 25 & $1.56(0.87,2.81)$ & 0.47 \\
\hline Combined & 31703 & 399 & $1.28(1.10,1.48)$ & 369 & $1.45(1.24,1.68)$ & 103 & $1.91(1.43,2.55)$ & 0.0012 \\
\hline
\end{tabular}

Shown are the number $(N)$ of controls and cases, the OR with a $95 \% \mathrm{Cl}$ and two-sided $P$-value for regressing the severity status on rs $7216389-T$ allele counts for the individual studies and the combined study populations.

a Onset of asthma at or younger than 18 years of age. $P$-values for heterogeneity between effect size in sample groups were $0.47,0.54$ and 0.24 for mild, moderate and severe asthma, respectively, in the combined analysis.

We then assessed the association of rs7216389-T with asthma, stratified by age at onset, in six additional sets of asthma patients: Australian, Dutch and Korean sample sets consisting of adult asthma cases, a UK sample set consisting of both adult and child asthma cases and two German sample sets of childhood asthma cases (Table 1). As in Iceland, the association of rs7216389-T with asthma was confined to the early-onset cases (OR $(95 \% \mathrm{CI})=1.33(1.21-1.46)$ for early-onset asthma compared with OR $(95 \% \mathrm{CI})=1.02 \quad(0.91-1.15)$ for adult-onset asthma) (Table 2, Supplementary Table 3). The effect of rs7216389-T in samples consisting of childhood asthma cases (OR $(95 \% \mathrm{CI})=1.30(1.13-1.50))$ was similar to that seen for adult cases with early-onset asthma (OR $(95 \% \mathrm{CI})=1.35(1.19-1.52))$.

Information on age at onset of asthma in years was available for Iceland, the United Kingdom, Germany II, Australia and Korea. To increase the resolution of the effect of age at onset of asthma, we further assessed the association with rs7216389-T by grouping cases into 2-year bins $(0-1,2-3,4-5,6-7$ years and so on, up to over 70 years). Nominally significantly increased risk was observed for the age bins $0-1,2-3,4-5,14-15$ and 16-17 years (Figure 1, Supplementary Table 4). Subsequent analysis of larger bins (0-5, 6-13, 14-17 years) within the early-onset-of-asthma group showed strong association for the intervals $0-5$ and $14-17$ years at onset $(\mathrm{OR}(95 \% \mathrm{CI})=1.51$ (1.31-1.73), $P=6.89 \cdot 10^{-9}$ and $\mathrm{OR}(95 \% \mathrm{CI})=1.71 \quad(1.39-2.11)$,
$P=5.47 \cdot 10^{-9}$, respectively), whereas a weaker association was observed in the age interval $6-13$ years $(\mathrm{OR} \quad(95 \% \mathrm{CI})=1.17 \quad(1.01-1.35)$, $P=0.035$ ). The Dutch and Germany I samples were only classified as early or late onset and were therefore not included in the higherresolution age at onset analysis.

Given that the strength of association with rs7216389-T was primarily observed in early-onset cases, we further examined whether rs7216389-T associated with sex, asthma severity, atopy status and serum IgE levels within early- and adult-onset cases. Asthma severity scoring was available for the Icelandic, Australian and UK cases. In the combined analysis of the early-onset cases, rs7216389-T correlated positively with asthma severity $(P=0.0012$, Table 3$)$, with the effect in the same direction for all three groups, although not significant in the UK sample set. No association with severity was observed among adult-onset asthma cases $(P=0.75$, Supplementary Table 6$)$. No association was observed with $\operatorname{IgE}(P=0.35)$ or atopy status $(P=0.55)$ (Supplementary Table 5), and no difference was observed between male and female cases $(P=0.60)$.

Restricting the analysis to early-onset asthma cases, we then looked for signals not accounted for by rs7216389-T. For this purpose, association with 14 additional SNPs was assessed in the Icelandic, Australian, UK and German samples, in which each marker was tested by conditioning on the observed association with each of the other 
markers (Supplementary Table 7). None of the markers remained significant after adjusting for the association with rs7216389-T and accounting for the number of SNPs tested.

\section{Correlation between 17q21 sequence variants and gene expression} levels

The SNP rs7216389 is located within the GSDMB gene (Supplementary Figure 1). We assessed the association of rs7216389 with gene expression levels by probes representing 29 refseq genes and 3 mRNA sequences located $540 \mathrm{~kb}$ upstream and downstream of s7216389 in white blood cell RNA obtained from Icelandic individuals $(N=743){ }^{29}$ The strongest association of rs7216389 was seen with the expression of GSDMB and $O R M D L 3$ genes $\left(P=2.3 \cdot 10^{-38}\right.$ and $P=8.8 \cdot 10^{-58}$, respectively, Table 4 and Supplementary Table 8 ). The expression of GSDMB and ORMDL3 is correlated in white blood cells (observed correlation r0.71; Supplementary Figure 2). We verified the association of rs7216389 with expression levels by RT-PCR TaqMan assays for GSDMB and ORMDL3 on the same RNA used for array experiments and subsequently in additional RNA samples as well, which were derived from PBMCs $(N=182)$ and EBV-transformed B-lymphocytes $(N=186)$ (data not shown). Finally, we assessed whether sequence variants other than rs7216389 associated more strongly with the expression of ORMDL3 and GSDMB genes. After adjusting for the effect of rs7216389 and correcting for multiple testing, 11 of the 14 markers tested remained significantly associated with ORMDL3 expression levels, whereas 9 markers remained significantly associated with GSDMB levels $(P<0.0036=0.05 / 14$, Table 4$)$.

\section{DISCUSSION}

This study confirms the previously reported genome-wide association of a sequence variant at $17 \mathrm{q} 21$ with asthma. We also show, using a large sample including six sets of European and one of East-Asian ancestry, that the variant is predominantly a risk factor for early-onset asthma, whereas its association with adult-onset asthma is absent. The risk associated with rs7216389-T was significant at $0-5$ years and 14-17 years of age at the onset of asthma. The original discovery ${ }^{6}$ and subsequent studies ${ }^{7-10}$ reported that the $17 \mathrm{q} 21$ locus is primarily associated with pediatric asthma. In this study, we confirm and further extend these findings by showing that association is not only confined to cases with onset in early childhood but also to cases with onset in adolescence.

Asthma is a phenotypically heterogeneous disease, with great variation in symptom severity and frequency and in the age at onset. One of the strengths of this multicenter study is that it includes both child and adult asthma cases with early or adult onset with variable disease severity, allowing for assessment of the risk contributed by the $17 \mathrm{q} 21$ variant in multiple asthma subphenotypes. The bimodal pattern of association with rs7216389-T with respect to age at onset in all sample sets combined, as also observed for individual sample sets (data not shown), suggests that the 17q21 locus not only confers increased risk for developing asthma in early childhood as has been reported, ${ }^{8}$ but also presents a substantial risk for onset of asthma in adolescence.

In this study, all cases were diagnosed by a physician using standardized international and national criteria. Although it cannot be excluded that ascertainment criteria influenced the results, we observed a consistent association of rs7216389-T with early-onset asthma in all samples sets, but not with adult-onset asthma in any sample set. It is unlikely that recall bias or differences in ascertainment would result in the bimodal pattern observed. Self-reported year of asthma onset by adults was accurate in cases with a clinical diagnosis of asthma followed up after 10 years $^{34}$ and in the longitudinal European Community Respiratory Health Survey. ${ }^{35}$ High concordance for reported age at onset with a self-administered questionnaire

Table 4 Association with early onset asthma and the expression of the ORMDL3 and GSDMB genes in white blood cells ( $N=743$ ) for 15 markers at $17 q 21$, both unadjusted and adjusted for the effect of rs7216389-T

\begin{tabular}{|c|c|c|c|c|c|c|c|c|c|}
\hline \multirow[b]{2}{*}{$S N P$} & \multirow[b]{2}{*}{ Allele } & \multirow[b]{2}{*}{ Position $^{a}$} & \multirow[b]{2}{*}{ OR } & \multicolumn{2}{|c|}{$\begin{array}{l}\text { Association with early onset asthma } \\
\qquad \mathrm{P} \text {-value }\end{array}$} & \multicolumn{2}{|c|}{$\begin{array}{c}\text { Association with ORMDL3 expression } \\
\text { P-value }\end{array}$} & \multicolumn{2}{|c|}{$\begin{array}{l}\text { Association with GSDMB expression } \\
\qquad \mathrm{P} \text {-value }\end{array}$} \\
\hline & & & & Unadjusted & rs7216389 adj. & Unadjusted & rs7216389 adj. & Unadjusted & rs7216389 adj. \\
\hline rs2941504 & $A$ & 35084426 & 1.29 & $1.2 \cdot 10^{-8}$ & 0.032 & $3.6 \cdot 10^{-21}$ & 0.66 & $1.2 \cdot 10^{-11}$ & 0.31 \\
\hline rs907091 & $\mathrm{T}$ & 35175268 & 1.34 & $1.7 \cdot 10^{-12}$ & 0.47 & $2.6 \cdot 10^{-60}$ & $3.4 \cdot 10^{-5}$ & $1.4 \cdot 10^{-42}$ & $8.3 \cdot 10^{-6}$ \\
\hline rs907092 & $\mathrm{G}$ & 35175785 & 1.34 & $1.7 \cdot 10^{-12}$ & 0.18 & $5 \cdot 10^{-63}$ & $1.5 \cdot 10^{-10}$ & $5.1 \cdot 10^{-36}$ & 0.0012 \\
\hline rs9303277 & $\mathrm{C}$ & 35229995 & 1.37 & $2.8 \cdot 10^{-14}$ & 0.09 & $8.3 \cdot 10^{-61}$ & $2.1 \cdot 10^{-5}$ & $1.1 \cdot 10^{-41}$ & $6.1 \cdot 10^{-5}$ \\
\hline rs3816470 & $\mathrm{T}$ & 35239327 & 1.37 & $4.3 \cdot 10^{-14}$ & 0.031 & $1.3 \cdot 10^{-58}$ & $3.5 \cdot 10^{-5}$ & $2.4 \cdot 10^{-42}$ & $4.7 \cdot 10^{-6}$ \\
\hline rs1 1557467 & G & 35282160 & 1.35 & $3.1 \cdot 10^{-13}$ & 0.45 & $5.4 \cdot 10^{-62}$ & $5.8 \cdot 10^{-6}$ & $1.5 \cdot 10^{-42}$ & $1.2 \cdot 10^{-5}$ \\
\hline rs8067378 & $A$ & 35304874 & 1.35 & $4.6 \cdot 10^{-13}$ & 0.72 & $5.4 \cdot 10^{-62}$ & $5.8 \cdot 10^{-6}$ & $1.5 \cdot 10^{-42}$ & $1.2 \cdot 10^{-5}$ \\
\hline rs2305480 & C & 35315722 & 1.36 & $1.3 \cdot 10^{-13}$ & 0.12 & $9.1 \cdot 10^{-65}$ & $2.3 \cdot 10^{-10}$ & $5.5 \cdot 10^{-37}$ & 0.0030 \\
\hline rs2305479 & $G$ & 35315743 & 1.37 & $1.7 \cdot 10^{-14}$ & 0.11 & $7.7 \cdot 10^{-61}$ & 0.00016 & $6.8 \cdot 10^{-43}$ & $1.8 \cdot 10^{-6}$ \\
\hline rs2290400 & A & 35319766 & 1.38 & $6.7 \cdot 10^{-15}$ & 0.018 & $1.2 \cdot 10^{-60}$ & 0.00029 & $1.3 \cdot 10^{-41}$ & $5.6 \cdot 10^{-5}$ \\
\hline rs7216389 & $\mathrm{T}$ & 35323475 & 1.36 & $5.1 \cdot 10^{-14}$ & - & $8.8 \cdot 10^{-58}$ & - & $2.3 \cdot 10^{-38}$ & - \\
\hline rs4795405 & $\mathrm{C}$ & 35341943 & 1.37 & $6.6 \cdot 10^{-14}$ & 0.018 & $5.8 \cdot 10^{-60}$ & $1.8 \cdot 10^{-8}$ & $1.2 \cdot 10^{-33}$ & 0.016 \\
\hline rs1007654 & $\mathrm{G}$ & 35364880 & 1.32 & $6.9 \cdot 10^{-10}$ & 0.040 & $1.3 \cdot 10^{-28}$ & 0.1031 & $9.4 \cdot 10^{-16}$ & 0.84 \\
\hline rs3902025 & $A$ & 35372780 & 1.32 & $1.0 \cdot 10^{-10}$ & 0.050 & $8.6 \cdot 10^{-39}$ & 0.00030 & $5.5 \cdot 10^{-22}$ & 0.15 \\
\hline rs3894194 & $\mathrm{T}$ & 35375519 & 1.32 & $3.9 \cdot 10^{-11}$ & 0.078 & $8.9 \cdot 10^{-34}$ & 0.03775 & $5.4 \cdot 10^{-20}$ & 0.59 \\
\hline
\end{tabular}

The calculated association for early onset asthma is obtained by combining the study populations (Iceland, Australia, UK, Germany I and Germany II) using a Mantel-Haenszel model. Log-transformed expression levels of ORMDL3 and GSDMB were corrected for age and sex. Correlation between MLR and allele count was performed treating the corrected log expression level as the response variable and the allele as the explanatory variable for each of the 15 markers. Shown are the OR and two-sided $P$-values, unadjusted and adjusted when the association is tested conditional on the observed association to SNP rs7216389.

aBase pair position March 2006 human reference sequence (NCBI Build 36). 
and face-to-face interview was also noted in the Epidemiological Study on Genetics and Environment of Asthma. ${ }^{8}$ Imperfect recall may dilute the age bins in which the $17 q 21$ variant has an effect, but is unlikely to shift these ages from early childhood to adolescence. The same would apply for differences in ascertainment for age at onset. Most individuals diagnosed with asthma in the first two decades of life have had recurrent wheezing episodes in early childhood, ${ }^{36,37}$ suggesting that the disease process may start years before diagnosis. Thus, cases with onset in adolescence may have had asthma-related symptoms such as bronchial hyperresponsiveness or wheezing episodes during childhood, or alternatively, changes in environmental exposure during adolescence could trigger asthma onset in susceptible individuals. The adolescent period is associated with changes in social behavior, such as initiation of smoking. Bouzigon et al ${ }^{8}$ reported that $17 \mathrm{q} 21$ variants were associated with substantially increased risk for asthma in children exposed to environmental smoke, and regular smoking has been associated with increased risk of new-onset asthma in adolescents. ${ }^{38}$ Our study was neither sufficiently powered nor detailed enough with respect to information on smoking initiation and duration to examine the relation of smoking pattern and risk for asthma associated with rs7216389-T in 14-to17-year-old subjects.

rs7216389-T correlated with asthma severity in early onset in the three sample sets analyzed, but not with adult-onset cases. This is in line with results obtained from two recent studies that reported an association of the rs7216389 $\mathrm{T} / \mathrm{T}$ homozygote state with more frequent asthma exacerbations ${ }^{7,11}$ and hospitalizations. ${ }^{11}$ Asthma severity is a complex composite phenotype ${ }^{39}$ based on the frequency and severity of asthma symptoms, as well as on lung function and treatment response. ${ }^{25,26}$ Most asthma patients with mild-to-moderate asthma can adequately control symptoms with standard therapies, but an estimated $5-10 \%$ of patients with severe asthma are largely unresponsive to typical therapies, including corticosteroids. ${ }^{40}$ Disease severity contributes to a large portion of morbidity in asthma. ${ }^{41}$ Our demonstration that rs7216389-T associates with severity in early-onset asthma could contribute to an earlier identification of individuals at high risk of developing severe asthma, allowing for more appropriate therapy at an earlier stage. Moffatt et al ${ }^{6}$ demonstrated that rs7216389 correlates with the expression of ORMDL3, and suggested that rs7216389-T confers the risk of asthma by affecting the expression of the ORMDL3 gene. We confirmed the association of rs7216389-T with expression levels of ORMDL3, and extended this observation to its association with the expression of the GSDMB gene, in which rs7216389 is located. We also found that the expression of these genes is highly correlated, suggesting that they may be coregulated. Several sequence variants at $17 \mathrm{q} 21$ associating with asthma affect expression levels of both GSDMB and ORMDL3. We showed that the variants with the strongest association with asthma were different from those that were most strongly associated with the expression of ORMDL3 or $G S D M B$. Furthermore, we observed a significant residual association for many markers with expression levels of ORMDL3 or GSDMB after adjusting for the effect of rs7216389. This shows that an alteration of the expression levels of either of these genes is unlikely to constitute the sole mechanism by which $17 \mathrm{q} 21$ sequence variants contribute to asthma susceptibility.

Given the relatively strong LD at this locus, we examined key SNPs tagging the region for association with early-onset asthma. However, after adjusting for the effect of rs7216389-T, association with other markers was nonsignificant. Two recent studies found no significant association of rs7216389-T with asthma in African-American patients, ${ }^{10,42}$ which, given the difference in LD structure in European and African Americans, suggests that rs7216389-T is not the causal variant itself and that the causal variant is in lower LD with rs7216389-T in African Americans. Recently, other SNPs highly correlated with rs7216389 were found to be associated with Crohn's disease (rs2872507) ${ }^{43}$ and type I diabetes (rs2290400), ${ }^{44}$ indicating that sequence variants at the $17 \mathrm{q} 21$ locus may be involved in mechanisms regulating immune responses. Further studies focusing on both gene and protein expression in relevant tissues and/or appropriate asthma models with extended phenotypic and genetic refinement are warranted to elucidate the precise mechanisms by which the 17q21 sequence variation contributes to asthma.

In summary, in addition to robustly replicating sequence variants that associate with asthma at the 17q21 locus, our data demonstrate that they confer a different risk of development of asthma at early age compared with onset at adult age in cases of European and Asian ancestry. The 17q21 locus associated with disease severity among early-onset but not adult-onset cases. We further show that the sequence variants showing the strongest association with asthma are different from those best associated with the expression of ORMDL3 or GSDMB. This suggests that the contribution of this locus to asthma susceptibility is unlikely to operate only through the expression of ORMDL3 or GSDMB genes, and that other mechanisms, or genes, contribute to the association.

\section{CONFLICT OF INTEREST}

The authors declare no conflict of interest.

\section{ACKNOWLEDGEMENTS}

We are grateful to all the participating patients and control populations described in this paper. We also thank personnel at the deCODE core facilities and members of clinical centers for valuable contribution, in particular Ms Jo Horn, Ms Amber Bullock, Guido Fischer, Liane Thaller, Margret Bahnweg Bettina Wunderlich, Christine Braig and J Britton for access to phenotypes from the Gedling population (Nottingham).

1 Cookson WOC: Asthma genetics. Chest 2002; 121: 7S-13S.

2 Ober C, Hoffjan S: Asthma genetics 2006: the long and winding road to gene discovery. Genes Immun 2006; 7: 95-100.

3 Tattersfield AE, Knox AJ, Britton JR, Hall IP: Asthma. Lancet 2002; 360: 1313-1322.

4 Hoffjan S, Nicolae D, Ober C: Association studies for asthma and atopic diseases: a comprehensive review of the literature. Respir Res 2003; 4: 14.

5 Zhang J, Pare PD, Sandford AJ: Recent advances in asthma genetics. Respir Res 2008; 9: 4.

6 Moffatt MF, Kabesch M, Liang L et al: Genetic variants regulating ORMDL3 expression contribute to the risk of childhood asthma. Nature 2007; 448: 470-473.

7 Bisgaard $\mathrm{H}$, Bonnelykke K, Sleiman PMA et al: ORMDL3 associated gene variants are associated with asthma and exacerbations but not atopy in early childhood. Am J Respir Crit Care Med 2008, 2009; 179: 179-185.

8 Bouzigon $\mathrm{E}$, Corda $\mathrm{E}$, Aschard $\mathrm{H}$ et al: Effect of $17 \mathrm{q} 21$ variants and smoking exposure in early-onset asthma. N Engl J Med 2008; 359: 1985-1994.

9 Hirota T, Harada M, Sakashita M et al: Genetic polymorphism regulating ORM1-like 3 (Saccharomyces cerevisiae) expression is associated with childhood atopic asthma in a Japanese population. J Allergy Clin Immunol 2008; 121: 769-770.

10 Sleiman PMA, Annaiah K, Imielinski M et al: ORMDL3 variants associated with asthma susceptibility in North Americans of European ancestry. J Allergy Clin Immunol 2008; 122: 1225-1227.

11 Tavendale R, Macgregor DF, Mukhopadhyay S, Palmer CN: A polymorphism controlling ORMDL3 expression is associated with asthma that is poorly controlled by current medications. J Allergy Clin Immunol 2008; 121: 860-863.

12 Madore AM, Tremblay K, Hudson TJ, Laprise C: Replication of an association between 17q21 SNPs and asthma in a French-Canadian familial collection. Hum Genet 2008; 123: 93-95.

13 Altmuller J, Seidel C, Lee YA et al: Phenotypic and genetic heterogeneity in a genomewide linkage study of asthma families. BMC Pulm Med 2005; 5: 1.

14 Britton J, Pavord I, Richards $\mathrm{K}$ et al: Factors influencing the occurrence of airway hyperreactivity in the general population: the importance of atopy and airway calibre. Eur Respir J 1994; 7: 881-887.

15 Hakonarson $\mathrm{H}$, Bjornsdottir US, Halapi E et al: A major susceptibility gene for asthma maps to chromosome 14q24. Am J Hum Genet 2002; 71: 483-491. 
16 Heinzmann A, Jerkic S-P, Ganter K et al: Association study of the IL13 variant Arg110GIn in atopic diseases and juvenile idiopathic arthritis. J Allergy Clin Immunol 2003; 112: 735.

17 Kedda MA, Shi J, Duffy D et al: Characterization of two polymorphisms in the leukotriene $\mathrm{C} 4$ synthase gene in an Australian population of subjects with mild, moderate, and severe asthma. J Allergy Clin Immunol 2004; 113: 889-895.

$18 \mathrm{Kim}$ Y, Park CS, Shin HD et al: A promoter nucleotide variant of the dendritic cellspecific DCNP1 associates with serum IgE levels specific for dust mite allergens among the Korean asthmatics. Genes Immun 2007; 8: 369-378.

19 Koppelman G, Stine O, Xu J et al: Genome-wide search for atopy susceptibility genes in Dutch families with asthma. J Allergy Clin Immunol 2002; 109: 498-506.

20 Postma DS, Meyers DA, Jongepier H, Howard TD, Koppelman GH, Bleecker ER: Genomewide screen for pulmonary function in 200 families ascertained for asthma. Am J Respir Crit Care Med 2005; 172: 446-452.

21 van Diemen CC, Postma DS, Vonk JM, Bruinenberg M, Schouten JP, Boezen HM: A disintegrin and metalloprotease 33 polymorphisms and lung function decline in the general population. Am J Respir Crit Care Med 2005; 172: 329-333.

22 Wheatley AP, Bolland DJ, Hewitt JE, Dewar JC, Hall IP: Identification of the autoantigen SART-1 as a candidate gene for the development of atopy. Hum Mol Genet 2002; 11: 2143-2146.

23 Wjst M, Fischer G, Immervoll T et al: A genome-wide search for linkage to asthma German Asthma Genetics Group. Genomics 1999; 58: 1-8.

24 Gudbjartsson DF, Bjornsdottir US, Halapi $\mathrm{E}$ et al: Sequence variants affecting eosinophil numbers associate with asthma and myocardial infarction. Nat Genet 2009; 41: 342-347.

25 Department of Health and Human Services PHS, National US and Institutes of Health, National Heart, Lung, and Blood Institute. Expert Panel Report 2: Guidelines for the Diagnosis and Management of Asthma. National Institutes of Health: Washington, 1997.

26 The Global Initiative for Asthma (GINA): Global Strategy for Asthma Management and Prevention. The Global Initiative for Asthma (GINA), 2005; http//www.ginaasthma.com.

27 Council ANA: Asthma Management Handbook. Council ANA, 2002: Melbourne, Australia.

28 British guidelines on the management of asthma. Thorax 2003; 58: i1-i94.

29 Emilsson V, Thorleifsson G, Zhang B et al: Genetics of gene expression and its effect on disease. Nature 2008; 452: 423-428.
30 Amundadottir LT, Sulem P, Gudmundsson J et al: A common variant associated with prostate cancer in European and African populations. Nat Genet 2006; 38: 652-658.

31 Hakonarson $\mathrm{H}$, Bjornsdottir US, Halapi $\mathrm{E}$ et al: Profiling of genes expressed in peripheral blood mononuclear cells predicts glucocorticoid sensitivity in asthma patients. Proc Natl Acad Sci USA 2005; 102: 14789-14794.

32 Gretarsdottir S, Sveinbjornsdottir S, Jonsson $\mathrm{HH}$ et al: Localization of a susceptibility gene for common forms of stroke to 5q12. Am J Hum Genet 2002; 70: 593-603.

33 Mantel N, Haenszel W: Statistical aspects of the analysis of data from retrospective studies of disease. J Natl Cancer Inst 1959; 22: 719-748.

34 Toren K, Palmqvist M, Lowhagen O, Balder B, Tunsater A: Self-reported asthma was biased in relation to disease severity while reported year of asthma onset was accurate. J Clin Epidemiol 2006; 59: 90-93.

35 Pattaro C, Locatelli F, Sunyer J, de Marco R: Using the age at onset may increase the reliability of Iongitudinal asthma assessment. J Clin Epidemiol 2007; 60: 704-711.

36 Morgan WJ, Stern DA, Sherrill DL et al: Outcome of asthma and wheezing in the first 6 years of life: follow-up through adolescence. Am J Respir Crit Care Med 2005; 172: 1253-1258.

37 Stern DA, Morgan WJ, Halonen M, Wright AL, Martinez FD: Wheezing and bronchial hyper-responsiveness in early childhood as predictors of newly diagnosed asthma in early adulthood: a longitudinal birth-cohort study. The Lancet 2008; 372: 1058.

38 Gilliland FD, Islam T, Berhane $\mathrm{K}$ et al: Regular smoking and asthma incidence in adolescents. Am J Respir Crit Care Med 2006; 174: 1094-1100.

39 Wenzel SE, Busse WW: Severe asthma: lessons from the severe asthma research program. J Allergy Clin Immunol 2007; 119: 14.

40 Moore WC, Bleecker ER, Curran-Everett D et al: Characterization of the severe asthma phenotype by the national heart, lung, and blood institute's severe asthma research program. J Allergy Clin Immunol 2007; 119: 405-413.

41 Barnes PJ, Jonsson B, Klim JB: The costs of asthma. Eur Respir J 1996; 9: 636-642.

42 Galanter J, Choudhry S, Eng C et al: ORMDL3 gene is associated with asthma in three ethnically diverse populations. Am J Respir Crit Care Med 2008; 177: 1194-1200.

43 Barrett JC, Hansoul S, Nicolae DL et al: Genome-wide association defines more than 30 distinct susceptibility loci for Crohn's disease. Nat Genet 2008; 40: 955-962.

44 Barrett JC, Clayton DG, Concannon P et al: Genome-wide association study and meta-analysis find that over 40 loci affect risk of type 1 diabetes. Nat Genet 2009; 41: 703-707.

Supplementary Information accompanies the paper on European Journal of Human Genetics website (http://www.nature.com/ejhg) 